separately the right and left generators. Refer the pairs of right generators to the pairs of points where they meet a conic of the surface.

Then we are to prove that if $p_{1}, p_{2}, p_{\mathbf{s}}$ are pairs of points on a conic, and the pair harmonic with $p_{2}$ and $p_{3}$ be $p_{1}^{\prime}$, and the pair harmonic with $p_{1}$ and $p_{1}^{\prime}$ be $p_{1}^{\prime \prime}$, then $p_{1}^{\prime \prime}, p_{2}^{\prime \prime}$, $p_{3}{ }^{\prime \prime}$ are in involution. In other words, replacing the pairs of points by the lines through them, we are to prove that a triangle and its polar triangle as to a conic are in perspective, a known elementary proposition. The ten pairs of points on the conic are in fact the points in which the lines of the configuration $10_{3}$, the intersection of a plane with a complete five point cut the conic to which it is self polar.

Thus as three pairs of points on a conic determine the configuration of ten pairs of points, so three quadrilaterals in the surface determine a configuration of ten quadrilaterals, and replacing each quadrilateral by its diagonal pair we have the configuration of Professor Study.

HAVERFORD COLLEGE, November, 1898.

\title{
BESSEL'S FUNCTIONS.
}

Einleitung in die Theorie der Bessel'schen Funktionen, von Professor J. H. Graf und Dr. E. Gubler, Erstes Heft: Funktionen erster Art. Bern, 1898, Wyss \& Co.

Is the last few years the study of Bessel's functions has been enriched by many contributions, the subject generalized for the complex variable, and the premises much more carefully defined.

Among English speaking investigators the interest seems to lie chiefly in two directions, theoretical consideration of the differential equation, and practical applications, numerical tabulation, etc.

The book under review does not favor any particular side but may be said to put the most emphasis upon the definite integral. Numerical tabulation is not touched upon in the part which has appeared; the remaining part, soon to appear, may have a chapter devoted to this purpose.

The first feature is a carefully arranged historic introduction, which contains an extensive list of books and memoirs 
on the subject. The origin of the Bessel differential equation is ascribed to Riccati ; after briefly citing various physical applications the history of the development of Kepler's problem is given from the time of its origin to the definition,

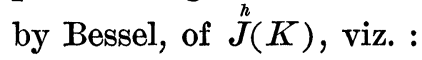

$$
2 \pi \stackrel{h}{J}(K)=\int_{0}^{2 \pi} \cos (h e-K \sin e) d e .
$$

The problemi tself is then stated and solved "according to Bessel," though the authors have skillfully modernized the solution by regarding the variable as complex throughout, determining coefficients by repeated use of Cauchy's theorem, and plotting multiple valued functions on a Riemann's surface. The chapter is closed by expressing these functions as definite integrals and infinite series, the latter leading up to the formal definition

$$
\stackrel{a}{J}(x)=\sum_{\lambda=0}^{\infty}(-1)^{\lambda} \frac{\left(\frac{x}{2}\right)^{a+2 \lambda}}{\lambda ! \Gamma(a+\lambda+1)}
$$

with which the treatment really begins.

The evaluations of $\bar{J}^{3 / 6}(x), \bar{J}^{3 / 3}(x)$ are first obtained, then the relations which exist when $a$ is an integer $n$,

$\bar{J}^{n}(x)=(-1)^{n} \stackrel{n}{J}(x), \stackrel{n}{J}(-x)=\bar{J}^{n}(x), \vec{J}^{-n}(-x)=\stackrel{n}{J}(x)$.

By means of differential operators the usual relations are easily established; from the form

$$
\stackrel{a}{J}(x)=(a-1) \frac{2}{x}^{a-1} J(x)-{ }^{a-2}(J x)
$$

the general form

$$
\stackrel{a}{J}(x)=A_{m}^{a-m} J(x)-A_{m-1}^{a-m-1} J(x)
$$

is neatly proved by induction, wherein

$$
A_{m}=\sum_{\lambda=0}^{E}\left(\frac{m+1}{2}\right)(-1)^{\lambda}\left(\begin{array}{c}
m-\lambda \\
\lambda
\end{array}\right) \frac{\Gamma(a-\lambda)}{\Gamma(a-m+\lambda)}\left(\frac{2}{x}\right)^{m-2 \lambda}
$$

After expressing $\stackrel{m+3 / 6}{J}(x)$ by means of a trigonometric series the differential equation is obtained in an original way. 
$\bar{J}^{a}(x)$ is shown to be a second particular integral ; but $\stackrel{a}{J}(x)$, $\bar{J}(x)$ not being independent when $a$ is an integer (see (2)), Schläfli's form of the second integral is introduced

$$
\stackrel{a}{K}(x)=\cot a \pi \stackrel{a}{J}(x)-\frac{1}{\sin a \pi} \cdot \bar{J}^{a}(x)
$$

as the complementary function; it is developed in a series by the method already used for $\stackrel{a}{J}(x)$, and some pretty problems in evaluating indeterminate forms are neatly solved. The complementary function is shown to satisfy the relations already found for $\stackrel{a}{J}(x)$.

The conjugate functions $\stackrel{a}{P}(x), \stackrel{a}{Q}(x)$ are now defined, and I ommel's and Weber's formulas derived. The chapter closes with a discussion of the effect which would be produced on the function by rotating $x$ through $m$ half circles in the positive half plane. The discussion is extended to apply to $\stackrel{a}{K}(x), \stackrel{a}{P}(x), \stackrel{a}{Q}(x)$.

This chapter is clearly and forcibly written; the results are obtained after an easy and natural process, without the long maze of formulas frequently found in the older treatments.

The second chapter of thirty-three pages treats of the representation of Bessel's functions by means of definite integrals. For this purpose the generalized $\Gamma$-function

$$
\frac{1}{\Gamma(a)}=\frac{1}{2 \pi i} \int e^{u} u^{-a} d u
$$

is used as the starting point, and this value of $\Gamma(a)$ is introduced into the first definition (1) of $\stackrel{a}{J}(x)$, which reduces to

$$
\stackrel{a}{J}(x)=\frac{1}{2 \pi i}\left(\frac{x}{2}\right)^{a} \int e^{u-\frac{x^{2}}{4}} u^{-a-1} d u=\frac{1}{2 \pi i} \int e^{\frac{x}{2}\left(t-\frac{1}{t}\right)} t^{-a-1} d t .
$$

Although not all of the results are new, this chapter exhibits a great deal of Graf's own work in his successful use of the "Schleifenintegral," which may be said to be almost characteristic of his researches.*

The related functions are then treated in the same way, and several results are obtained by changing the form of the

* $C f$., especially, his Theorie der $\Gamma$-Funktionen and Auswertung bestimmter Integrale, and the dissertations of Schenkel and Eggenberger. 
path of integration. The successive steps of this process are so clear that a student familiar with the elements of complex integration would have his ideas greatly extended and systematized by reading the chapter.

Finally, the same functions are expressed as integrals of simpler functions, the path of integration being a "Doppelumlauf" around the point $i x$ and extending to infinity parallel to the negative part of the axis of reals. The complex variable is designated by $x$ or by $u$, and the real component by $t$. Hyperbolic functions are distinguished from trigonometric ones by the use of the German letters, thus

$\cosh x$ by $\cos x$, $\sinh x$ by fin $x$.

One difficulty of this paragraph is to know just what end is in view. The reader is easily and directly carried on from form to form, often without distinctly knowing why, except, as the heading of the chapter suggests, to start from the first definition, and deduce the various definite integrals which define the same function.

The third chapter discusses the problem of determining the value of $\stackrel{a}{J}(x)$ for large values of $x$. The starting point is

$$
\stackrel{a}{P}(x)=e^{i(a-1) \frac{\pi}{2}} \frac{(2 x)^{-a}}{\Gamma\left(\frac{1}{2}\right) \Gamma\left(\frac{1}{2}+a\right)} \int_{-N+i x}^{i x} e^{u}\left(u^{2}+x^{2}\right)^{a-1 / 2} d u
$$

in which $N$ is large, hence $u$ approaches a real negative value at the lower limit. When $x \doteq \infty$, and the integration is reduced to straight line integrals by Cauchy's theorem,

$$
\stackrel{a}{P}(x) \doteq \frac{e^{i\left(x-\frac{\pi}{4}\right)}}{\sqrt{2 \pi x}} ; \text { similarly } Q \doteq \frac{e^{-i\left(x-\frac{\pi}{4}\right)}}{\sqrt{2 \pi x}},
$$

$Q$ always being derived from $P$ by changing the sign of $i$. By means of the relations between $P, Q, J, K$, when $x \doteq \infty$,

$$
\begin{aligned}
& \stackrel{a}{J}(x) \doteq \sqrt{\frac{2}{\pi x}} \cos \left[x-\left(a+\frac{1}{2}\right) \frac{\pi}{2}\right], \\
& \stackrel{a}{K}(x) \doteq \sqrt{\frac{2}{\pi x}} \sin \left(x-\left(a+\frac{1}{2}\right) \frac{\pi}{2}\right) .
\end{aligned}
$$

Corresponding simple forms are obtained for $P, Q, J, K$, when $x$ replaced by $\pm i x, e^{ \pm \frac{i \pi}{2}} x, e^{\mp \frac{3 \pi i}{2}} x$. 
The same problem is then considered from another standpoint; a semi-convergent series is obtained for $\stackrel{a}{P}(x)$ and it is proved that the remainder after $n$ terms is numerically less than the $(n+1)$ th term. *

A third method is applied to the case in which $x$ is positive. Starting with the formula

$$
\stackrel{a}{J}(x)=\frac{1}{2 \pi i} \int e^{\frac{x}{-}\left(t-\frac{1}{t}\right)} t^{-a-1} d t
$$

a transformation is made, and the new path of integration exhibited on a two leaved surface. One formidable expression for $J(x)$ is produced, consisting of a trigonometric series with $\Gamma$-functions as coefficients, a remainder term, and two double integrals. This is the only place in the work in which a double integral is employed.

An appendix is added to this chapter in which the problem of evaluating

$$
\lim _{a \doteq \infty} \stackrel{a}{J} J(x)
$$

is discussed. Three cases are considered, according as $x$ is less than, equal to, or greater than unity. Duhamel's and Poisson's forms are derived, and a new form

$$
\lim _{a \doteq \infty} a^{\frac{1}{3}} \stackrel{a}{J}(a)=\frac{\Gamma\left(\frac{1}{3}\right)}{\pi 2^{\frac{2}{3}} 3^{\frac{1}{6}}}
$$

as dividing line between the two.

The fourth chapter obtains the derivative of $\stackrel{a}{J}(x)$ with regard to the parameter, and has several theorems on the roots of $\stackrel{a}{J}(x)=0$. The parameter is always regarded as real. Excluding the case in which $a$ is a negative integer, $\left[\frac{x}{2}\right]^{-a} \stackrel{a}{J}(x)=0$ can never have a double root; when $a>1$, th $\theta$ roots are always real; when $-2<a<-1$, two rootsare imaginary ; and two more roots become imaginary for every negative integer greater than $\alpha$, as $a$ dimishes towards $-\infty$. When $-1<a$, the positive $\operatorname{roots}\left(\alpha_{s}\right)$ of ${ }^{a} \bar{J}^{-1}(x),\left(\beta_{s}\right)$ of ${ }^{a} J(x)$ and $\left(\gamma_{s}\right)$ of $\stackrel{a+1}{J}(x)$ satisfy the relations

* Cf. Rayleigh, Theory of Sound, for a similar remark for $\stackrel{a}{J}(x)$. 


$$
0<\alpha_{1}<\beta_{1}<\gamma_{1}<\alpha_{2}<\beta_{2}<\gamma_{2}<\cdots<\alpha_{s}<\beta_{8}<\gamma_{8} .
$$

These numerous important results are clearly and precisely obtained in ten pages, with intervening steps sufficiently indicated to enable one to read the matter readily.

The last chapter discusses the development of functions with Bessel's functions for coefficients. After discussing the convergence of a particular series, the general form is assumed

$$
f(x)=\sum_{\lambda=1}^{\infty} A_{\lambda} \cdot \vec{a}\left(B_{\lambda} x\right) \quad\left[\stackrel{a}{J}\left(B_{\lambda}\right)=0\right]
$$

and the $A_{\lambda}$ are to be obtained according to the process employed in Fourier's series by means of the theorem

$$
\int_{0}^{1} \stackrel{a}{J}\left(B_{\kappa} x\right) \stackrel{a}{J}\left(B_{\lambda} x\right) d x=0 .
$$

The result (Schlömilch's theorem) is

$$
f(x)=\sum_{m=1}^{\infty} \int_{0}^{1} \frac{2 t \stackrel{a}{J}\left(B_{m} x\right) \stackrel{a}{J}\left(B_{m} t\right) f(t)}{[a+1} d t .
$$

A discussion of several pages concerning the convergence of this series closes the volume; although the details are not hard to follow, it is by no means clear just what conditions must be fulfilled to have the series represent the function.

Copious references are given throughout, and a list of some fifty memoirs is added, though most results previously obtained were derived in quite a different way.

On the whole the book has a fresh and original appearance; Chapters I and IV especially are, in the reviewer's opinion, the most readable presentation extant of the subject treated.

CORNELL UNIVERSITY,

VIRgIL SNYder. October 18, 1898. 